\title{
La construcción de inferencias en la comprensión lectora: una investigación correlacional
}

\section{Building inferences in reading comprehension: $\mathrm{A}$ correlational study}

\author{
Itzi Paulina Medina Jiménez*1 \\ itzipau12@gmail.com \\ Carlos González Di Pierro** \\ carlos.dipierro@umich.mx \\ * Universidad Autónoma de Querétaro, México \\ **Universidad Michoacana de San Nicolás de Hidalgo, México
}

\section{Resumen:}

El objetivo del estudio consiste en identificar, a partir de una prueba de comprensión lectora off-line de dos tipos textuales, si existe correlación entre aprobar una materia de lectura (en español como lengua materna) en escolaridad superior (licenciatura) y una mejoría en la comprensión lectora respecto al nivel previo a la asignatura. El instrumento fue pilotado hasta llegar a su versión final, donde el texto técnico exige inferencias proposicionales y el texto humanístico requiere inferencias pragmáticas. El análisis de los resultados se realizó en dos fases: primero, se evaluó el nivel de comprensión lectora derivado de cada uno de los textos por separado y después fueron comparados ambos estratos para saber si los alumnos que aprobaron la asignatura tienen en realidad un nivel de comprensión más alto que quienes no la cursaron. La discusión reveló que no existe correlación entre el desempeño en la prueba técnica y el desempeño en la prueba humanística. Tampoco existe correlación entre aprobar la asigna-

\begin{abstract}
:
This piece of research aims to identify if there is any correlation between passing a reading university course (with Spanish as a first language) and an improvement in reading comprehension, compared with the reading level obtained before attending the classes. After testing our reading comprehension tool among different pilot groups, we reach the final version, which has two different texts: the first one is a technical text, which requires readers to make propositional inferences, and the second one is a humanistic text, which requires readers to make pragmatic inferences. The analysis of the results was undertaken in two phases: first, we evaluated the reading comprehension level of each text, and second, we compared both levels to know if students who had passed had a higher reading comprehension level than students who had never taken this reading course. The discussion showed that there is not any correlation between technical test performance and humanistic test performance; neither is there a correlation
\end{abstract}

1 Dirección para correspondencia (correspondence address):

Paulina Medina Jiménez. Calle Trabajadores de Agricultura n. 280, Colonia FOVISSSTE Morelos, C.P. 58120, Morelia, Michoacán (México). 
La construcción de inferencias en la comprensión lectora: una investigación correlacional

Itzi Paulina Medina Jiménez y Carlos González Di Pierro

tura y el nivel de comprensión lectora, ni de un texto técnico ni de uno inferencial; de hecho, el nivel de comprensión lectora no presenta mejorías significativas. En conclusión, esto implica que, tras cursar una materia de comprensión lectora, los estudiantes siguen siendo incapaces de realizar las operaciones mentales necesarias para llegar hasta el nivel de representación que el tipo de texto exige tanto en el nivel proposicional como en el pragmático.

\section{Palabras clave:}

Lectura; comprensión; inferencia; operaciones mentales; tipos textuales; discurso. between passing the subject and the level of reading comprehension. Moreover, reading comprehension did not show any significant improvement. In sum, after taking the entire course, students are still unable to perform the mental operations needed to reach the representation level (propositional or pragmatical) expected to comprehend both text types.

\section{Key words:}

Reading; understanding; inferences; mental operations; text tipology; discourse.

\section{Résumé:}

L'objectif de ce travail est d'identifier s'il existe une corrélation entre la réussite du cours de Lecture (Espagnol en tant que langue maternelle) au niveau universitaire et I'amélioration de la compréhension des textes, comparée au niveau obtenu avant de commencer le cours, en utilisant une épreuve de compréhension de lecture off-line de deux types textuels différents. L'outil qui sert à réaliser cette recherche a été soumis à des essais pour la perfectionner jusqu'à sa version finale, dans laquelle le texte technique exige de faire des inférences propositionnelles et le texte des sciences humaines exige de faire des inférences pragmatiques. L'analyse des résultats a été réalisée en deux parties. Premièrement, le niveau de compréhension écrite issue de chacun des textes a été évalué séparément. Deuxièmement, les deux niveaux ont été comparés pour savoir si les élèves admis avaient un niveau plus élevé en compréhension écrite par rapport à ceux qui n'ont pas suivi le cours. La comparaison des résultats a dévoilé qu'il n’y a pas de corrélation entre la performance à l'épreuve technique et la performance à l'épreuve des sciences humaines. Les données montrent de même qu'il n'y a pas de corrélation entre la réussite de l'année universitaire et le niveau de compréhension de texte, quel que soit le type de texte. En effet, le niveau de compréhension de lecture ne montre aucune amélioration significative. En conclusion, les résultats de la recherche démontrent qu'après avoir suivi le cours de Lecture à l'université, les apprenants ne sont pas capables d'effectuer les opérations mentales nécessaires pour atteindre le niveau de représentation exigé par le type de texte, soit au niveau propositionnel, soit au niveau pragmatique.

\section{Mots clés:}

Lecture; compréhension; inférences; opérations mentales; typologie textuelle; discours.

Fecha de recepción: 22-10-2020

Fecha de aceptación: 26-12-2020 


\section{Introducción}

La presente investigación se realizó como parte del proyecto "Didáctica de las operaciones mentales que intervienen en el proceso de enseñanza y aprendizaje", Ilevado a cabo por el grupo de investigación UMS0049 "Estudios Lingüísticos y Literarios", reconocido por la Secretaría de Educación Pública de México a través del Programa para el Desarrollo Profesional Docente (PRODEP) entre los años 2015 y 2018. Fue financiada con fondos del Estado de Michoacán al formar parte del cuerpo de proyectos de la Coordinación de la Investigación Científica de la Universidad Michoacana en México.

Con el propósito de poder determinar la correlación existente entre cursar una asignatura cuya finalidad es la de desarrollar la habilidad de comprensión lectora en estudiantes de ingeniería y la mejora en el desempeño de dicha competencia, se llevó a cabo un estudio experimental con el diseño de un instrumento a través del cual se pudiesen medir estos factores.

Tomando como punto de partida el modelo de comprensión lectora de van Dijk y Kintsch (1983), la caracterización de los procesos inferenciales de Chikalanga (1992) y la conceptualización de la comprensión como operación mental inherente al proceso de aprendizaje (Tuffanelli, 2000), se concibió un estudio con dos pruebas: un texto que se denominó técnico, que requería para su comprensión de que se realizaran inferencias proposicionales, y un texto al que se le Ilamó humanístico, mismo que exigía inferencias pragmáticas para responder adecuadamente.

Se establece aquí en primer término el marco conceptual utilizado, después se describe el estudio empírico enfatizando el proceso de creación del instrumento en virtud de que una parte importante del estudio consistió en la propuesta para elaborar pruebas que determinen si estudiantes universitarios de ciencias exactas son capaces de comprender tanto aquellos textos que son necesarios para su formación académica como otro tipo de textos que son parte de su experiencia personal. Esta medición es en función de que puedan o no realizar inferencias de tipo léxico, lógico proposicional o pragmático.

En la discusión y resultados se puede apreciar que no se encontró una correlación entre el desempeño de ambas pruebas y tampoco hay correlación entre cursar la asignatura y el aumento del nivel de comprensión lectora, ni con el texto técnico de baja complejidad inferencial, ni con el humanístico donde se requieren inferencias de mayor nivel. 


\section{Marco Teórico}

La manera como se concibe el acto de leer y la comprensión lectora con todo lo que le subyace para efectos de establecer una perspectiva didáctica, se desprende directamente, sobre todo para el nivel educativo superior que es el caso de la investigación que nos ocupa, de tener clara la manera en que se quiere entender la actividad lectora; es decir, de los múltiples enfoques que existen y se siguen desarrollando para abordar este importante proceso, es necesario primero definir cuál será más adecuado en el ámbito específico docente e investigador.

Para establecer el marco teórico y conceptual se hizo un breve recorrido por tres perspectivas fundamentales: la lectura desde la teoría literaria; desde la sociología de la lectura y como proceso cognitivo desde la psicología. El enfoque de una teoría literaria pensada lato sensu para efectos de nuestro trabajo, se estudia a través del pensamiento de Gadamer (1993) y sus desarrollos hermenéuticos, la existencia de un horizonte del lector y un horizonte del texto, la construcción intersubjetiva que hace que lo leído adquiera diferentes sentidos; de igual manera las posturas de Ricoeur (1999) en el sentido de que dichas ideas gadamerianas de los "horizontes" pretenden el acercamiento entre texto y lector cuya pretensión es reconocer el otro horizonte en el propio, sin perder de vista que el texto es fijo, se orienta desde la producción del mismo. Finalmente se analizaron algunas de las ideas de Ingarden (1998) el cual enlista cuatro estratos en los que estaría dividida toda obra literaria, los cuales, el entrar en juego entre sí, permiten al lector concretar y apropiarse de la obra: estrato lingüístico, estrato semántico, estrato de los objetos representados y estrato de los objetos esquematizados.

Una segunda perspectiva teórica que posibilita estudiar el proceso lector y su comprensión es la que se engloba como sociología de la lectura, cuyo interés se centra en los entornos en que se produce y se recibe la obra, las categorías sociales que intervienen, los ámbitos y contextos que hacen que se fomente la lectura, incluso la importancia que tiene el Estado en todo el acto social de leer, así como otros temas provenientes de la teoría sociológica y que pueden encuadrarse en el proceso lector. Según Cros (1993), hay una multiplicidad de criterios de estudio dentro de la sociología de la lectura, los cuales en ocasiones se ven mermados por el propio rechazo que tienen escritores y lectores para ser captados de manera sociológica, creando una natural resistencia hacia el cuerpo social. 
El tercer enfoque teórico-conceptual que se analiza para decidir cómo encaminar el presente estudio, es el de la psicología de la lectura, específicamente la concepción del acto lector como proceso cognitivo, implicando operaciones mentales que se desprenden de dicho proceso y, siempre dentro de la misma perspectiva, la comprensión. En el siguiente apartado, se presentan las ideas y conceptos utilizados desde esta teoría para los efectos conducentes de este trabajo.

\section{Leer como proceso cognitivo desde la psicología}

En virtud de que esta investigación está circunscrita, como se verá un poco más adelante, en el estudio del proceso de comprensión lectora de estudiantes de ingeniería y concretamente en la capacidad inferencial que se mide a través de un instrumento diseñado para ello, se tomó el modelo de van Dijk y Kintsch (1983) como base para profundizar en los conceptos más necesarios e importantes del proceso de comprensión lectora; junto con esto, se emplearon posteriores desarrollos teóricos derivados de tal perspectiva. Dichos autores parten de la premisa de que la lectura es una actividad que se mueve en el plano mental, vinculándose constantemente con ciertos aspectos del mundo circundante; por ende, ponen énfasis en el discurso y la relación existente con los procesos cognitivos, mismos que se relacionan a su vez con elementos sociales y culturales propios de cada sujeto particular. Es decir, explican la lectura como un proceso integral y de acción.

En su modelo interactivo de comprensión lectora, van Dijk y Kintsch (1983) estudian las representaciones semánticas de la mente, más que la actividad neuronal, es decir que se trata de un modelo simbolista. Se destaca la multidimensionalidad del mismo ya que reúne procesos cognitivos y componentes socio-culturales pero sin perder de vista al texto como elemento que está hecho para guiar hacia ciertos significados durante la lectura (van Dijk y Kintsch, 1983). Hay un equilibrio en cuanto a la importancia que se da a texto y lector, y es de esta idea que se desprende esta concepción interactiva de la lectura que se materializa por un doble movimiento: la lectura como proceso top-down, que está protagonizado por el lector como ente que vierte sus experiencias y conocimientos para conocer al texto; y la lectura como proceso bottom-up, en el cual tiene mayor importancia o protagonismo el texto, pues contiene elementos para lograr ser comprendido. En esta visión interactiva, 
la lectura es bidireccional, de arriba hacia abajo y de abajo hacia arriba porque tanto lector como texto van construyendo de la mano los significados necesarios para la adecuada comprensión.

Los citados autores partieron del supuesto de que la comprensión lectora consiste en fijar en la mente una imagen coherente de lo que se lee, es decir, construir una representación mental del contenido textual (van Dijk y Kintsch, 1983). Para lograr esa coherencia, se pasa por tres niveles de representación semántica: el código lingüístico o código de superficie, el texto base y el modelo situacional. En esencia, en el código de superficie se realiza el proceso de descodificación, mientras que en la base textual se da significado a las proposiciones mediante la construcción de inferencias simples, aunque estos niveles son interactivos, se alternan en un devenir constante. Y con lo anterior, se logra construir el tercer nivel, en el cual se pone en marcha el proceso inferencial más complejo introduciendo micro y macro estructuras en un modelo situacional, donde el texto con su información gramatical y estructural, y el lector con su conocimiento gramatical y del mundo, interactúan y funcionan de manera conjunta para así alcanzar la comprensión (van Dijk y Kintsch, 1983).

\section{El proceso inferencial}

En el proceso de comprensión lectora resulta de crucial importancia el concepto de conocimiento del mundo, conocimiento compartido o simplemente el saber del mundo. Cuenca y Hilferty (2006) señalan que en la comunicación hay una parte denotativa, que se identifica con las cuestiones gramaticales, y otra connotativa que es aquella contenida en el concepto que tenemos de la realidad. Al momento de leer, el proceso inferencial que proviene de introducir a nuestros esquemas mentales la información del mundo, resulta de suma importancia ya que desde que inicia la lectura, se requiere de la anticipación del sentido del nivel más bajo para poder establecer la cohesión y la coherencia local, en primer término, y conforme continúa avanzando la lectura, se va anticipando también y de manera constante el sentido macro del texto para así llegar a una coherencia global del mismo.

Para terminar de conceptualizar el proceso inferencial es imprescindible hacer una caracterización de la comprensión como operación mental esencial del proceso de enseñanza y aprendizaje. Según Morin (1999) 
existen dos tipos de comprensión: una comprensión intelectual, objetiva y una comprensión humana, intersubjetiva. La primera de ellas se manifiesta en la inteligibilidad y se allega del pensamiento lógico, mientras que la segunda, con todo y que puede transitar dicha inteligibilidad, de hecho la sobrepasa debido a que implica un grado de empatía con el objeto, poniendo en juego el conocimiento de un sujeto hacia otro. Tal comprensión subjetiva va más allá del pensamiento lógico para llegar a un pensamiento narrativo, entendiendo lo narrativo como el medio para poder construir la identidad personal y colectiva, es decir, el pensamiento del mundo, la experiencia de la vida, la alteridad.

En este sentido, para efectos de esta investigación, resulta imprescindible abordar un tema que en términos del complejo mundo de la comprensión se viene realizando desde hace varios siglos: la distinción entre ciencias duras, exactas o naturales y las ciencias humanas o sociales, en muchas ocasiones incluso excluidas de su carácter de ciencias para ser denominadas simplemente "humanidades". Según Tuffanelli (2010), las ciencias duras generan un saber nomológico mientras que las humanas generan un saber idiográfico, lo cual resulta que las primeras se preocupan más por explicar y las segundas por comprender, distinción muy similar a la que se expuso anteriormente a partir de Morín (1999).

Sin embargo, se ha constatado que asumir que ambos tipos de saberes tienen posiciones extremas y disociadas entre sí, es equivocado debido a que implicaría asegurar que no puede haber puntos de intersección entre unas y otras ciencias, y que dentro de este distanciamiento los seres humanos no serían capaces de explicar su entorno. A pesar de ello, en un país como México, donde se realizó este estudio, continúa imperando la creencia de que las ciencias humanas constituyen un terreno que se aleja de las ciencias que gozan del status de exactitud y comprobación experimental dura. Al ampliar la búsqueda conceptual de esta dicotomía, se sigue que cada tipo de ciencia inevitablemente debe reconocer no ser autosuficiente, sino que existe una interdependencia multidisciplinar cuando se quieren incrementar los horizontes académicos y la exploración del mundo desde ambas formas de comprensión.

Para concluir esta caracterización general del marco teórico-conceptual, hacemos un recorrido por la categorización realizada por Chikalanga (1992) respecto de las inferencias. En la primera fase de la construcción del instrumento de prueba, que se describirá más adelante, se parte del modelo de van Dijk y Kintsch (1983) para identificar los niveles 
de representación semántica (el código de superficie, la base textual y el modelo situacional); para la segunda parte se emplea la tipología de inferencias de Chikalanga (1992): inferencias léxicas, lógicas proposicionales y pragmáticas.

En primer lugar, las inferencias léxicas descodifican la parte más superficial de un texto, pueden reconocer significados de palabras, relaciones pronominales, reconocer grupos de relaciones intradiscursivas y someras "... regarded as 'textually' or 'scriptally' implicit" (Chikalanga, 1992, p. 704). En segundo lugar, las inferencias lógicas proposicionales surgen del carácter lógico del texto, permiten en primer término captar el contenido proposicional y se deducen como derivación lógica del contenido semántico del texto. El citado autor las divide en informativas y explicativas, donde las primeras se limitan a dar cuenta de aspectos concretos que se traducen en el "qué" de lo leído, mientras que las explicativas son las que muestran modos, razones y relaciones causaconsecuencia, es decir, el "cómo" y el "porqué" del contenido.

Finalmente, en tercer lugar se encuentran las inferencias pragmáticas que rebasan el nivel proposicional del discurso y se adentran en materia del significado en uso, la adecuación, cuestiones que se ponen en marcha con la intervención del conocimiento del mundo que posee el lector. Se trata de un nivel donde podemos obtener el sentido de lo que no está en el texto en sí, sino que se puede activar a partir de él por su vínculo con el exterior, de ahí que para llegar a este nivel inferencial los saberes y estrategias de lectura deben ser los suficientes. En las inferencias pragmáticas, Chikalanga (1992) hace una distinción en tres categorías: informativas, explicativas y evaluativas. Las dos primeras guardan semejanza con las inferencias proposicionales, pero se distinguen de ellas en que están basadas en un proceso bottom-up, del lector hacia el texto y en cambio, estas inferencias lógicas proposicionales basan su informe o explicación en un proceso top-down.

Enseguida se verá cómo en un segundo momento de la investigación se utilizaron estas dos teorías, la de van Dijk y Kintsch (1983) y la de Chikalanga (1992), haciendo coincidir cada tipo de inferencias con su nivel correspondiente de representación semántica: al código de superficie le corresponden las inferencias léxicas, a la base textual las inferencias lógicas preposicionales y al modelo situacional las inferencias pragmáticas. Esto permitió construir un instrumento que toma en cuenta los procesos inferenciales como parte de las operaciones mentales nece- 
sarias para construir en la mente del lector una representación semántica coherente con el contenido textual: esto es, una alta comprensión lectora, tal como se detalla en el siguiente apartado.

\section{Marco empírico}

El objetivo de esta investigación fue determinar si existe una correlación entre cursar una asignatura de comprensión lectora y el nivel de dicha habilidad; esto se traduce en demostrar si se incrementa la capacidad de construir inferencias una vez que el estudiantado ha concluido un curso de comprensión lectora. Por ello, se diseñó un instrumento off-line, en formato físico, que mide distintos niveles inferenciales en la lectura de dos tipos de textos que exigen distintos niveles de representación mental, además de que permite realizar un análisis de resultados por texto, por tipo de inferencia y por grupo de participantes (quienes tomaron el curso frente a quienes no lo hicieron). Con esta metodología, la variable de grupo pudo someterse a pruebas de correlación con la variable de nivel de comprensión lectora.

Una parte del instrumento se conforma por un texto técnico de física clásica, "Gravedad simulada" (Hewitt, 2007), con el cual se debe realizar un mapa conceptual; esta actividad requiere inferencias de orden inferior. La otra parte consta de un texto humanístico de análisis social, "¿Mundo homogéneo?" (Savater, 1997), acompañado de una batería de preguntas abiertas y de opción múltiple. Cada respuesta se clasifica en un tipo inferencial de distinto nivel, lo cual permite observar si la construcción de inferencias se realiza de modo más complejo después de la asignatura o no.

\section{Instrumento}

En una primera fase de construcción de esta prueba, se partió del modelo de van Dijk y Kintsch (1983) para identificar tres niveles de representación semántica: código de superficie (CS), base textual (BT) y modelo situacional (MS). Seguido de esto se localizó su emparejamiento con la tipología de inferencias de Chikalanga (1992): léxicas, lógicas proposicionales y pragmáticas.

Así, se hizo coincidir cada tipo de inferencia con un nivel de repre- 
sentación semántica: al CS le corresponden las inferencias léxicas, a la BT las inferencias lógicas proposicionales y al MS las inferencias pragmáticas, como se muestra en la Tabla 1.

Tabla 1

Niveles de representación y tipos de inferencias

\begin{tabular}{llll}
\hline $\begin{array}{l}\text { Nivel de representación } \\
\text { semántica }\end{array}$ & Tipo de inferencia & Subtipo de inferencia & Siglas \\
\hline Código de superficie & Léxica & ${ }^{*}$ No hay subtipo & $\mathrm{CS}$ \\
Base textual & Lógica proposicional & Informativa & $\mathrm{LPi}$ \\
& & Explicativa & $\mathrm{LPe}$ \\
Modelo situacional & Pragmática & Informativa & $\mathrm{Pi}$ \\
& & Explicativa & $\mathrm{Pe}$ \\
& & Evaluativa & $\mathrm{Pev}$ \\
\hline
\end{tabular}

Posteriormente, se asignó a cada nivel de inferencia un puntaje, donde una mayor proximidad al modelo situacional equivale a una mejor comprensión lectora, siendo las inferencias pragmáticas evaluativas (Pev) y pragmáticas explicativas (Pe) las más complejas y, por ende, la cumbre de la lectura pues implican la realización de todos los niveles inferiores. En el otro extremo, la menor complejidad es la de la inferencia léxica, que se mantiene en el código de superficie (CS), es decir, no implica la construcción de significado sino únicamente la decodificación de grafías; al ser un nivel incapaz de contribuir a la construcción de una imagen mental del texto, el CS tiene un puntaje de 0 . A partir de la base textual, las inferencias tienen puntajes ascendentes comenzando en 1 con las LPi, tal como se muestra en la Tabla 2.

Tabla 2

Puntaje por subtipo de inferencia

\begin{tabular}{cc}
\hline Subtipo de inferencia & Puntaje \\
\hline CS & 0 \\
$\mathrm{LPi}$ & 1 \\
$\mathrm{LPe}$ & 2 \\
$\mathrm{Pi}$ & 3 \\
$\mathrm{Pe}$ & 4 \\
$\mathrm{Pev}$ & 4 \\
\hline
\end{tabular}


La medición de la capacidad evaluativa de una lectura, compete en gran medida a las teorías de argumentación y no forma parte de esta investigación; esta es la razón para otorgar, sin distinción, el máximo valor tanto a las Pe como a las Pev, subtipos que implican la forma más compleja de construcción inferencial.

Con base en lo anterior, se diseñó una primera sección para la evaluación de lectura de un texto humanístico (Savater, 1997). Primero se lee un texto continuo de 611 palabras, sobre la sociedad ante la globalización, con un enfoque filosófico, de lenguaje divulgativo (periodístico más que teórico, como el propio autor señala en otras ediciones); luego se responde una batería de preguntas inferenciales. Se generaron reactivos de opción múltiple que restringen las respuestas a subtipos inferenciales específicos, cada uno con su respectivo puntaje; a la par, se diseñaron reactivos abiertos y se clasificaron las respuestas en una inferencia específica para otorgarle una puntuación.

En este punto, un cuestionamiento bastante natural hacia este diseño sería qué tanto se aísla la variable del conocimiento del mundo y si, entonces, se mide el procesamiento del texto o los saberes previos con los que cuenta el lector. Como en toda evaluación lectora, más aún, en cualquier prueba de construcción de inferencias, es imposible deshacerse de las vivencias y experiencias del lector; a lo que se puede aspirar es a disminuir el impacto de esta variable.

Para el caso de este instrumento, si el sujeto no posee los conocimientos de la realidad extratextual sobre los personajes y hechos nombrados en la prueba, o si no posee un dominio filosófico, esto no imposibilita la inferencia, ya que el lector puede apoyarse en la información que brinda el universo intratextual para echar a andar las inferencias lógicoproposicionales y las inferencias pragmáticas, lo cual lleva a un nivel de lectura alto.

Ahora bien, con la segunda y última sección del instrumento se evalúa la lectura de un texto técnico (Hewitt, 2007) a través de su conceptualización. Consta de la lectura de un texto discontinuo de 916 palabras, con un enfoque didáctico y lenguaje divulgativo, sobre gravedad simulada, un tema de física clásica a partir del cual se debe realizar un mapa conceptual que rescate el contenido principal y sus relaciones.

Como se mencionó en el marco teórico, ya que el conocimiento previo tiene un impacto en la comprensión de los textos escritos (van Dijk y Kintsch, 1983), lo ideal es aminorar el efecto de esta variable sobre los 
resultados. De ahí que la elección de este texto parta de tres fundamentos: objetivo del curso, área científica común y temática novedosa. Primero, la asignatura se propone la lectura de diversas clases de materiales escritos, por ello la necesidad de introducir dos tipos textuales. Segundo, es un material de física, área científica que compete a la formación de ambas ingenierías (Civil y Mecánica). Tercero, aborda una teoría que no se encuentra dentro de los planes oficiales para bachilleres en México, ni en el primer año de estas dos ingenierías, por lo que el tema resulta nuevo para todos los sujetos sin importar bachillerato ni licenciatura de procedencia. Así, el segundo y tercer punto colocan en igualdad de circunstancias a los participantes en cuanto al conocimiento previo del tema.

Acerca del nivel de complejidad, se consultó con programas y profesores para seleccionar un escrito de índole conceptual que posee una estructura y temática accesible para su grado escolar; de hecho, la editorial sugiere el libro para Bachillerato. El texto elegido es discontinuo (contiene tres figuras, un recuadro con datos extra y una autoevaluación conceptual) y de carácter divulgativo, pues responde a las necesidades de los materiales didácticos de ciencias duras: ejemplos prácticos, datos curiosos y elementos visuales que simplifiquen la comprensión. Con esta selección del texto, se logró simular una de tantas clases de lecturas con la que se enfrentan los estudiantes de ingeniería a lo largo de, por lo menos, sus primeros años de Licenciatura.

Congruentes con este tipo textual, la tarea de lectura consiste en seleccionar, suprimir, generalizar y jerarquizar la información mediante la producción de un mapa conceptual, organizador gráfico que frecuentemente se solicita como tarea desde el nivel escolar básico hasta el superior. Los mapas conceptuales son una herencia del pensamiento computacional, descritos a profundidad por primera vez por Cañas y Novak (2006) como parte de su diseño experimental para la creación de software educativo, basados en diversas teorías cognitivas que defienden el aprendizaje significativo en vez del memorístico. De este modo, en la presente investigación se asume al mapa conceptual como el producto gráfico que refleja la representación mental que se construyó a partir del texto escrito.

Partiendo de la importancia de los modelos, en el instrumento se brinda un ejemplo de la estructura de un mapa conceptual, únicamente con los globos en blanco y las líneas de enlace, con lo cual se previene que 
algún participante realice otro tipo de organizador gráfico. En cuanto a su valoración, se toman en cuenta distintas evidencias de inferencias de menor complejidad (léxicas y lógicas proposicionales informativas) que corresponden a cinco actividades mentales que se eligieron como primordiales en la composición de un mapa conceptual, esto a partir de las macrorreglas propuestas por van Dijk y Kintsch (1983) y la caracterización que desarrollan Cañas y Novak (2006).

En la Tabla 3 se observa la rúbrica del mapa conceptual: las cinco actividades mentales consideradas, sus ocho evidencias y sus puntajes. En primer lugar, eliminar información no esencial del mapa conceptual es evidencia de la actividad de supresión; esto obtiene un punto. En segundo lugar, incluir la información relevante evidencia la actividad de selección y obtiene un punto. Tercero, la ubicación adecuada entre los globos según su relación, el uso de líneas de conexión entre unos globos y otros, así como el empleo de palabras de conexión entre globos, son tres evidencias de la correcta puesta en relación entre las proposiciones que conforman el texto leído; cada evidencia amerita un punto.

En cuarto lugar, realizar el mapa de lo general a lo particular en el sentido vertical descendente, al igual que el número de niveles en que se distribuya la información, son dos rasgos que evidencian la actividad de jerarquización. Colocar la información más general en la parte superior y paulatinamente avanzar hacia lo particular en los niveles inferiores, vale un punto. Emplear únicamente un nivel luego del globo raíz obtiene cero puntos, pues esto implica un término y su definición (y no un el verdadero análisis conceptual que propone un mapa conceptual); emplear dos niveles obtiene un punto; tres niveles, dos puntos; y cuatro niveles o más, tres puntos. Por último, la elección del concepto del globo raíz (el título) es una evidencia de que la actividad de globalización se ha realizado con éxito, y obtiene un punto.

Tabla 3

Rúbrica de mapa conceptual

\begin{tabular}{ccc}
\hline Actividad & Cómo se evidencia & Puntaje \\
\hline Supresión & Eliminar información no esencial & 1 \\
Selección & Incluir información relevante & 1 \\
Relación & Posición entre globos según su relación & 1 \\
& Uso de líneas de conexión & 1 \\
& Uso de palabras de conexión & 1
\end{tabular}




\begin{tabular}{ccc}
\hline Actividad & Cómo se evidencia & Puntaje \\
\hline Jerarquización & $\begin{array}{c}\text { De lo general a lo particular } \\
\text { Niveles luego del globo raíz }\end{array}$ & 1 \\
& 1 nivel & 0 \\
& 2 niveles & 1 \\
& 3 niveles & 2 \\
Globalización & 4 niveles o más & 3 \\
& Concepto del globo raíz (título) & 1 \\
\hline
\end{tabular}

\section{Aplicación y muestra}

De esta forma, una vez diseñado y pilotado el instrumento, se procedió a su aplicación definitiva en formato físico. Después se seleccionaron las pruebas a analizar, de acuerdo a los criterios de inclusión previamente establecidos: fueron tomadas en cuenta aquellas contestadas en su totalidad, con respuesta abierta o de opción múltiple según indica el reactivo, con no más de una respuesta por reactivo, con el tipo de organizador gráfico solicitado, este último realizado con la información del texto técnico, no del humanístico, y, finalmente, solo se incluyeron aquellas en que el sujeto reportó al español como su lengua materna.

Así, la muestra quedó compuesta por 50 sujetos de segundo año de Licenciatura: 25 sin cursar la asignatura de comprensión lectora, provenientes de Ingeniería Civil, y 25 que cursaron la asignatura, provenientes de Ingeniería Mecánica, en un rango etario entre 18 y 22 años, siendo el español su lengua materna.

\section{Resultados y discusión}

Primero, se realizó una prueba T para identificar diferencias por grupo (quienes cursaron la asignatura y quienes no lo hicieron) en la comprensión de ambos textos. Se pretende localizar si el desempeño lector de los estudiantes es distinto después de tomar el curso. Los resultados de este método paramétrico se muestran a continuación en la Tabla 4. 
Tabla 4

Resultados de la prueba $T$

\begin{tabular}{|c|c|c|c|c|}
\hline Tipo textual & Varianza & $\begin{array}{c}\text { Significancia } \\
\text { (bilateral) }\end{array}$ & $\begin{array}{l}\text { Diferencia } \\
\text { de medias }\end{array}$ & $\begin{array}{l}\text { Diferencia de } \\
\text { error estándar }\end{array}$ \\
\hline \multirow[t]{2}{*}{ Texto técnico } & $\begin{array}{c}\text { Se asumen } \\
\text { varianzas iguales }\end{array}$ & .880 & .120 & .793 \\
\hline & $\begin{array}{c}\text { No se asumen } \\
\text { varianzas iguales }\end{array}$ & .880 & .120 & .793 \\
\hline \multirow[t]{2}{*}{$\begin{array}{l}\text { Texto } \\
\text { humanístico }\end{array}$} & $\begin{array}{c}\text { Se asumen } \\
\text { varianzas iguales }\end{array}$ & .017 & .440 & .988 \\
\hline & $\begin{array}{c}\text { No se asumen } \\
\text { varianzas iguales }\end{array}$ & .017 & .440 & .988 \\
\hline
\end{tabular}

Se puede observar que esta comparación de medias no arrojó una diferencia significativa, lo cual indica que cursar la asignatura no coincide con un cambio importante en la lectura entre un tipo textual y otro: ni en la construcción de inferencias, ni en la realización de actividades mentales para conceptualizar un texto, así que el resultado contradice la hipótesis planteada en esta investigación.

Tras esto, los datos se sometieron a una prueba de correlación bivariada para localizar si el cambio en los valores de alguna de las variables coincide con el aumento o disminución de otra. En la Tabla 5 se exponen los resultados.

Tabla 5

Resultados de la prueba de correlaciones

\begin{tabular}{ccccc}
\hline Variable & & Texto técnico & $\begin{array}{c}\text { Texto } \\
\text { humanístico }\end{array}$ & Grupo \\
\hline Texto técnico & Correlación de Pearson & 1 & .156 & -.022 \\
& Significancia bilateral & & .280 & .888 \\
Texto & Correlación de Pearson & .156 & 50 & 50 \\
humanístico & & & $-.336^{*}$ \\
& Significancia bilateral & .280 & & .017 \\
\multirow{5}{*}{ Grupo } & $\mathrm{N}$ & 50 & 50 & 50 \\
& Correlación de Pearson & -.022 & $-.336^{*}$ & 1 \\
& Significancia bilateral & .888 & .017 & \\
& $\mathrm{~N}$ & 50 & 50 & 50 \\
\hline
\end{tabular}


El resultado significativo (señalado con asterisco) muestra que existe una correlación negativa débil entre el desempeño del texto humanístico y haber tomado el curso; esto indica que si aumenta la variable de grupo, disminuye la variable textual. Dicho en otras palabras, una vez que se ha tomado el curso, la capacidad para construir inferencias disminuye, no por una relación de causalidad pero si por una de correlación. Si bien esto no permite asegurar que la asignatura cause un detrimento en la comprensión lectora, tampoco justifica una relación proporcional donde la asignatura coincida con una mejora en esta habilidad lingüística. Dado que la correlación es débil, se procede a un análisis del ajuste de los datos para dar una interpretación más adecuada de ellos.

En la Figura 1 observamos los histogramas donde se presentan los resultados de los conjuntos analizados. Se muestra que las medias de ambos grupos (estudiantes que tomaron el curso, en azul; estudiantes que no, en rojo) difieren entre sí por un valor de 2.7 pero su desviación estándar es mayor a tres en cada caso. Esto último sugiere que la diferencia entre un grupo y otro solamente es debida al error estadístico asociado al experimento; por lo tanto, la correlación expuesta en la tabla puede explicarse como una consecuencia del error estadístico.

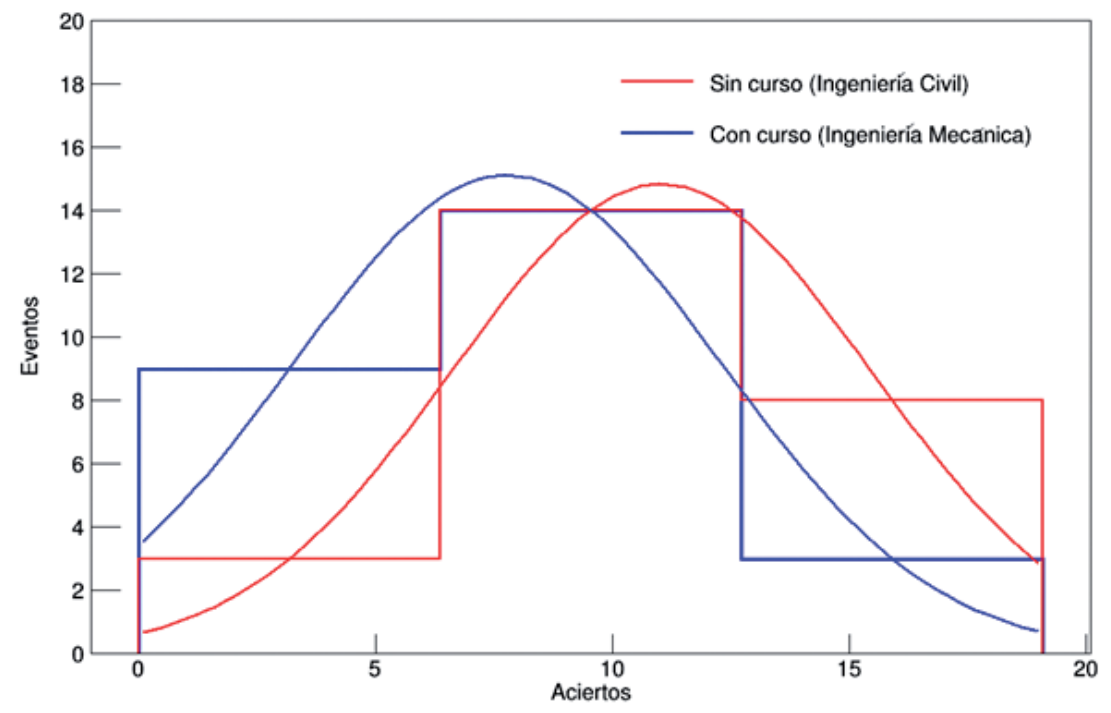

Figura 1. Histograma de aciertos en cada grupo.

Nota. El histograma presenta los aciertos en cada grupo de estudio, el grupo en rojo (Ingeniería Civil) no tomó el curso, mientras que el grupo en azul (Ingeniería Mecánica) sí lo tomó. 
Por otro lado, si ajustamos la distribución de datos mediante una función gaussiana, tal como se muestra en la Figura 1, el resultado es similar al histograma y nuevamente se observa un cambio en el máximo de la distribución pero que está dentro del error estadístico asociado. Además, la desviación estándar puede depender incluso de las variaciones existentes desde el inicio al interior de la muestra, esto es, las diferencias individuales que los sujetos que sí tomaron el curso ya presentaban antes del mismo, y que se desconocen al no contar con evaluaciones previas y sistemáticas de su nivel de comprensión lectora.

Para indagar al interior del instrumento se normalizan los datos en escala de 10 y se emplea una clasificación en tres niveles de lectura (Figuras 2 y Figura 3): bajo, para valores de 0 a 5.9; medio, de 6 a 7.9; y alto, en un rango de 8 a 10. Las fronteras de estos niveles responden a la necesidad de homologar los resultados con el sistema de evaluación que emplea la educación pública en México, donde la calificación mínima aprobatoria es 6 y se adopta como el límite inferior de suficiencia, mientras que una calificación superior a 8 suele considerarse sobresaliente. En ambos grupos y ambas pruebas el nivel imperante es el bajo, que es aún mayor en la prueba técnica del grupo que tomó el curso (Figura 3), aunque este incremento no es estadísticamente significativo. Cabe señalar que al unir los resultados de ambas pruebas, el promedio del desempeño en el instrumento es exactamente el mismo en ambos grupos: 4.7, una calificación reprobatoria.
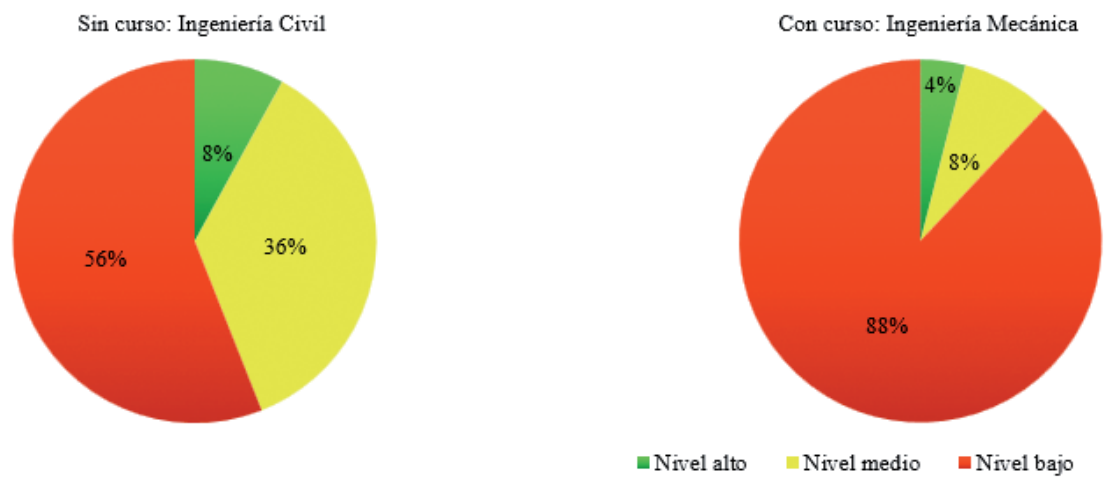

Figura 2. Niveles de lectura del texto humanístico por grupos. 
La construcción de inferencias en la comprensión lectora: una investigación correlacional

Itzi Paulina Medina Jiménez y Carlos González Di Pierro
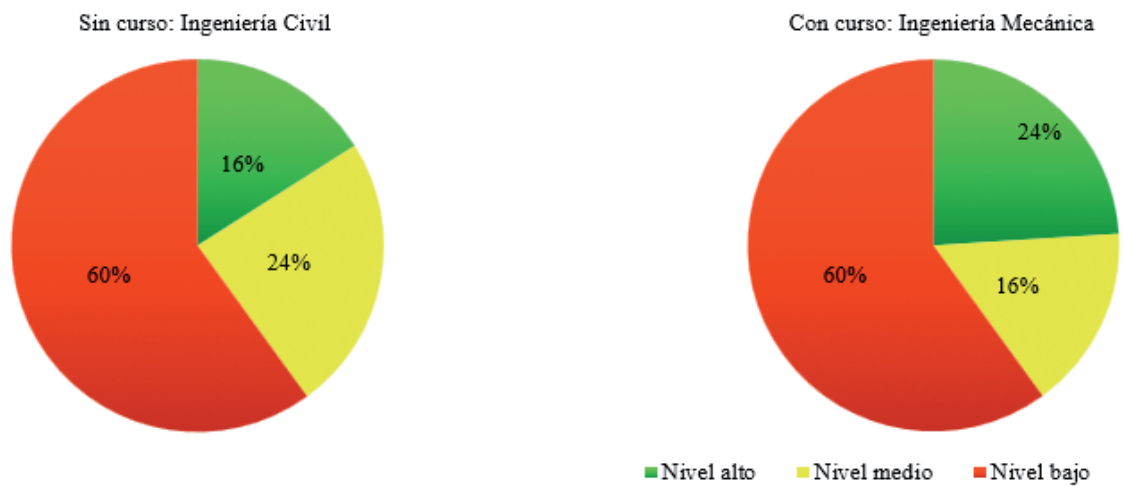

Figura 3. Niveles de lectura del texto técnico por grupos.

A continuación se detallan los resultados al interior de la prueba humanística. Para ambos grupos, en la primera mitad de reactivos cerrados la concentración de respuestas se ubica en niveles inferenciales de baja complejidad: los de las inferencias lógicas. Después, para la segunda mitad de preguntas cerradas, del grupo sin curso la mayoría de participantes (19 de 25) elige las respuestas de inferencias pragmáticas, al igual que el grupo que tomó el curso (16 sujetos de 25).

Pese a que el tipo de inferencias predominante es igual en ambos grupos (lógicas para la primera mitad de reactivos y pragmáticas para la segunda), cuando se procesan todos los reactivos cerrados, se observa que la aparición de cada subtipo inferencial es distinta de un grupo a otro, como se aprecia en la Tabla 6. Al nivel más alto de inferencias (pragmáticas evaluativas) Ilega más frecuentemente el grupo sin curso y a las pragmáticas explicativas Ilegan con más frecuencia quienes tomaron el curso.

Tabla 6

Texto humanístico: apariciones de cada tipo de inferencia en reactivos cerrados

\begin{tabular}{ccc}
\hline Inferencia & $\begin{array}{c}\% \text { Apariciones en } \\
\text { grupo sin curso }\end{array}$ & $\begin{array}{c}\% \text { Apariciones en } \\
\text { grupo con curso }\end{array}$ \\
\hline Lógica proposicional informativa & $20 \%$ & $20 \%$ \\
Lógica proposicional explicativa & $26 \%$ & $22.66 \%$ \\
Pragmática informativa & $3 \%$ & $2 \%$ \\
Pragmática explicativa & $5 \%$ & $8 \%$ \\
Pragmática evaluativa & $14 \%$ & $8 \%$ \\
\hline
\end{tabular}

Nota. El porcentaje muestra con qué frecuencia se elige cada tipo de inferencia sobre su máximo posible de apariciones, pues varía el número de respuestas ofrecidas para cada nivel inferencial. Por esto se reportan los datos en la misma escala sobre 100. 
En el caso de los reactivos abiertos, para ambos grupos de la muestra una tercera parte contiene más inferencias lógicas que de otro tipo; los otros dos tercios de los reactivos abiertos exponen una predominancia de inferencias pragmáticas. No obstante, la suma de apariciones por tipo (Tabla 7) muestra la frecuencia de cada clase y no su calidad, pues un factor común de las respuestas con inferencias pragmáticas es que a la par recuperan el código de superficie; esto indica que ambos grupos (más aun el que tomó el curso) son capaces de realizar actividades mentales pragmáticas pero sin independizarse del texto original. Se observó que junto a muchas de las inferencias pragmáticas existen valoraciones personales fuera de los límites temáticos del texto. Además, de la totalidad de reactivos de la prueba humanística, solo uno alcanza el nivel alto, esto en el grupo que no tomó el curso.

Tabla 7

Texto humanístico: apariciones de cada tipo de inferencia en reactivos abiertos

\begin{tabular}{ccc}
\hline Inferencia & $\begin{array}{c}\text { \% Apariciones en } \\
\text { grupo sin curso }\end{array}$ & $\begin{array}{c}\text { \%Apariciones en } \\
\text { grupo con curso }\end{array}$ \\
\hline Léxica & 18.67 & 36.00 \\
Lógica proposicional informativa & 25.33 & 28.00 \\
Lógica proposicional explicativa & 12.00 & 17.33 \\
Pragmática informativa & 22.67 & 6.67 \\
Pragmática explicativa & 18.67 & 12.00 \\
Pragmática evaluativa & 2.67 & 0 \\
\hline Total & 100.00 & 100.00 \\
\hline
\end{tabular}

Nota. El porcentaje muestra con qué frecuencia se presenta cada tipo de inferencia en reactivos abiertos, en escala sobre 100 , donde todos los tipos tienen la misma posibilidad de aparecer.

En cuanto a la prueba técnica los resultados son desalentadores para ambos grupos (Figura 4). La actividad de globalización es la única que se desempeña con un nivel alto; el resto ni siquiera alcanza el nivel medio. Esto se traduce en una deficiente capacidad para realizar las inferencias menos complejas: léxicas y lógicas informativas. 
La construcción de inferencias en la comprensión lectora: una investigación correlacional

Itzi Paulina Medina Jiménez y Carlos González Di Pierro

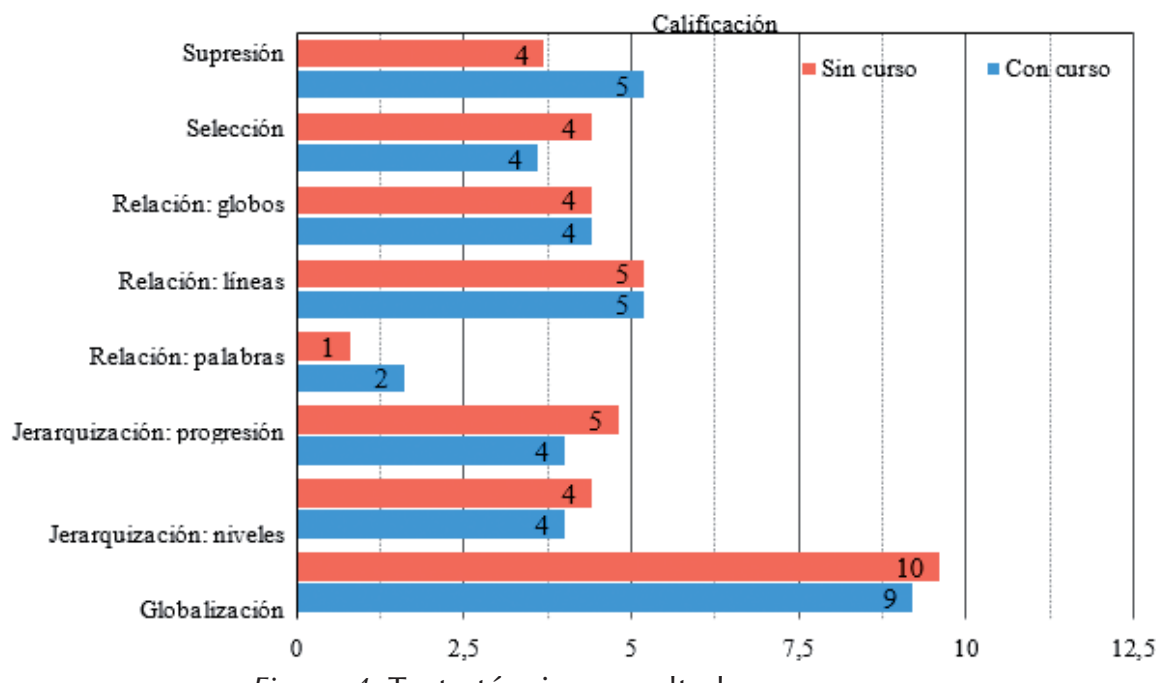

Figura 4. Texto técnico: resultados por grupo.

Para esta prueba sería deseable una segunda revisión por parte de otros evaluadores, pues daría más precisión sobre las evidencias de las actividades mentales implicadas en la conceptualización de un texto técnico. Así pues, este jueceo sería una posible mejora para el análisis de los resultados de dicha sección del instrumento.

\section{Conclusiones}

Podemos concluir al interpretar los resultados que:

- Apenas unos cuantos lectores logran calificaciones altas (9 o 10 ) en cualquiera de los dos textos: son alumnos arriba de la media.

- Además, de los estudiantes con desempeño alto en alguna de las dos pruebas, pocos consiguen el mismo resultado en ambas. Esto implica que las habilidades de construcción inferencial no están desarrolladas por igual en distintos tipos textuales.

- Si se promedian ambas pruebas por cada alumno, la mayoría obtiene un nivel bajo de lectura.

- En ambos tipos textuales persiste una lectura lineal y apegada al código de superficie: pocos estudiantes forman las relaciones al interior del discurso (para conceptualizarlo y construir inferencias) sin reproducir fragmentos textuales. 
- Los procesos inferenciales implicados en un texto humanístico representan gran dificultad para los alumnos de ambos grupos: algunos son incapaces de integrar saberes del mundo para construir inferencias pragmáticas y otros incorporan esos saberes deliberadamente, realizando inferencias muy alejadas del texto.

- Seleccionar, suprimir, relacionar, jerarquizar y englobar son actividades mentales que pocos alumnos pueden realizar bien como un conjunto; en consecuencia, no consiguen una conceptualización exitosa del texto.

- En la evaluación abierta del texto humanístico se introducen elementos intersubjetivos que podrían analizarse desde lo discursivo: exhortan a reflexionar, realizan juicios sociales y se identifican como parte de un grupo determinado o como actor en una relación de poder.

- Esta investigación abre la posibilidad de un análisis epistémico que vincule las prácticas lectoras específicas de sus carreras con su capacidad inferencial y de conceptualización.

- La calificación promedio en ambos grupos (4.7) y las pruebas estadísticas nos llevan a rechazar la hipótesis planteada al inicio de esta investigación: no se observa un incremento en la comprensión lectora de diversos tipos textuales (ni en lo conceptual ni en lo inferencial) tras cursar una asignatura que aborda esta habilidad lingüística.

\section{Referencias}

Cañas, A. y Novak, J. (2006). La teoría subyacente a los mapas conceptuales y a cómo construirlos. Reporte Técnico IHMC CmapTools 2006-01. Florida: Florida Institute for Human and Machine Cognition.

Chikalanga, I. (1992). A Suggested Taxonomy of Inferences for the Reading Teacher. Reading a Foreign Languaje, 8 (2), 697-709 pp.

Cros, E. (1993). Sociología de la literatura. En M. Angenot, M. et al., Teoría literaria (145171 pp.). México: Siglo XXI.

Cuenca, J. y Hilferty, J. (2006). Introducción a la lingüística cognitiva. Barcelona: Ariel.

Gadamer, H. (1993). Verdad y método. Salamanca: Sígueme.

Hewitt, P. (2007). Física conceptual. México: Pearson Educación.

Ingarden, R. (1998). La obra de arte literaria. México: Alfaguara.

Morín, E. (1999). Los siete saberes necesarios para la educación del futuro. París: Organización de las Naciones Unidas para la Educación, la Ciencia y la Cultura. 
La construcción de inferencias en la comprensión lectora: una investigación correlacional

itzi Paulina Medina Jiménez y Carlos González Dı Pierro

Ricoeur, P. (1999). Teoría de la interpretación: discurso y excedente de sentido. México: Siglo XXI.

Savater, F. (18 de mayo de 1997). ¿Mundo homogéneo?. El país semanal, p. 10.

Tuffanelli, L. (2010). Comprender. ¿Qué es? ¿Cómo funciona?. Madrid: Narcea-Ministerio de Educación.

van Dijk, T., \& Kintsch, W. (1983). Strategies of discourse comprehension. New York: Academic Press. 